\title{
Novel CenterCross and MultiCross devices for the treatment of infrainguinal chronic total occlusions: initial single-centre experience
}

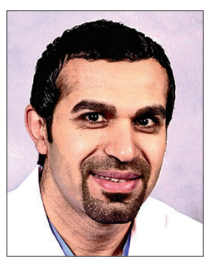

Shwan Jalal ${ }^{1 *}$, MD; Thomas A. Lalonde ${ }^{1}$, MD; Hiroshi Yamasaki ${ }^{1}$, MD; Andrew Boshara ${ }^{1}$, MD; Howard S. Rosman ${ }^{1}$, MD; Rajendra H. Mehta², MD, MS; Thomas P. Davis', MD

1. St. John Hospital and Medical Center, Detroit, MI, USA; 2. Duke Clinical Research Institute, Durham, NC, USA

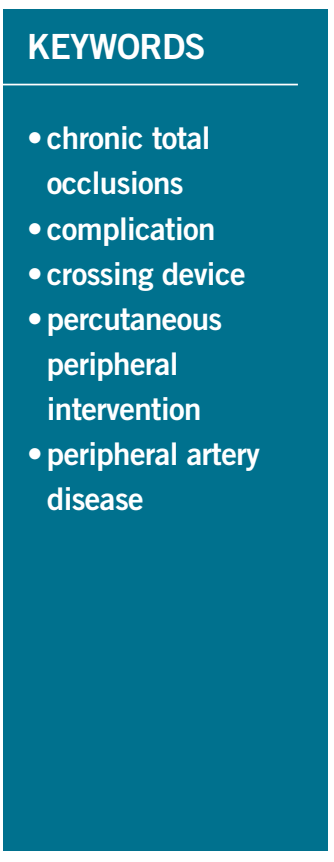

\begin{abstract}
Aims: Our aim was to evaluate the effectiveness and safety of CenterCross/MultiCross devices to facilitate the crossing of chronic total occlusions in peripheral arteries.

Methods and results: This was a single-centre study in which 53 consecutive patients who were not amenable to initial attempts at crossing using standard guidewires underwent an attempt to recanalise chronically occluded infrainguinal peripheral arteries with MultiCross/CenterCross devices. The primary endpoint of interest was the ability to advance the guidewire beyond the chronic total occlusion (CTO) lesions with the use of these devices. Safety endpoints were freedom from bleeding, distal embolisation and vessel perforation, dissection or need for emergent surgical intervention. Popliteal artery and below lesions were the most commonly treated, comprising $89 \%$ of the total. The CTO lesions were crossed successfully in $92.4 \%$ of the cases within a relatively short time (5.5 \pm 3.5 minutes). There was no bleeding, dissection or need for emergent surgery and $98.1 \%$ and $96.2 \%$ of the patients were free from distal embolisation and perforations, respectively.
\end{abstract}

Conclusions: Our study demonstrated that MultiCross and CenterCross were effective and safe for recanalisation of peripheral CTO lesions which were not amenable to conventional guidewires. Further study is required to define the role of these novel devices in the treatment of complex lesions, particularly CTOs in patients with peripheral arterial disease.

*Corresponding author: St. John Hospital \& Medical Center, 22101 Moross Road, 2nd Floor VEP, Detroit, MI, 48236, USA.

E-mail: Shwan.jalal@stjohn.org 


\section{Abbreviations}

CTO chronic total occlusion

PAD peripheral arterial disease

TASC TransAtlantic Inter-Society Consensus

\section{Introduction}

Peripheral arterial disease (PAD) affects about six to 12 million people in the United States of America and is associated with significant morbidity and mortality ${ }^{1}$. The symptoms of PAD include intermittent claudication, rest pain, tissue necrosis, ulceration, limb loss and death ${ }^{2}$. Up to $20 \%$ of the population of the USA above 75 years of age have some features of PAD. This prevalence is likely to increase with the aging of the general population $^{3}$.

Surgical bypass and/or surgical endarterectomy have been the main therapeutic options for alleviating symptoms, preventing limb loss and improving quality of life of patients with severe $\mathrm{PAD}^{3}$. In fact, bypass surgery has been endorsed as the preferred treatment for CTO lesions by TransAtlantic Inter-Society Consensus recommendations (TASC II) ${ }^{4}$. However, surgery is associated with high morbidity and mortality, particularly as many patients with PAD have increased comorbid conditions, including (amongst others) concomitant coronary artery and cerebrovascular disease $^{3,5}$. Endovascular treatment strategies, which are less invasive than bypass surgery, have evolved over the past 10 years and have increasingly become the revascularisation methods of choice for patients with severe PAD $^{5}$. The advantages of endovascular therapies over peripheral bypass surgery are rapid recovery and an early return to daily activities, less morbidity, shorter hospital stay and lower costs ${ }^{3,5}$.

However, the "Achilles heel" of endovascular therapeutic procedures remains chronic total occlusion (CTO), which represents about $20-40 \%$ of lesions in patients with symptomatic $\mathrm{PAD}^{1}$. Not only do these procedures for CTO require significant time, radiation and contrast use, but also, more importantly, they are associated with suboptimal procedural success rates and greater complications ${ }^{2}$. Failure to recanalise CTO occurs in $20 \%$ of cases: such procedures are not uncommonly associated with higher rates of complications such as arterial dissection and/or perforation, distal embolisation and bleeding ${ }^{6}$. These shortcomings have resulted in increasing interest and investment from operators as well as industry, not only in evaluating new strategies and therapies, but also in developing new technology to increase procedural success rates and decrease complications. As a result, there has been a proliferation of strategies and devices designed to improve the procedural success and outcomes in these patients ${ }^{7}$.

\section{Editorial, see page 971}

One such recent innovation to facilitate crossing of CTO in PAD patients is the arrival of the CenterCross ${ }^{\mathrm{TM}} / \mathrm{MultiCross}{ }^{\mathrm{TM}}$ devices (Roxwood Medical, Inc., Redwood City, CA, USA). We describe the initial experience with the use of these devices for the treatment of CTO at a single tertiary care centre.

\section{Methods \\ PATIENT POPULATION}

Between August 2014 and January 2015, 53 patients who were not amenable to initial attempts at standard guidewire crossing underwent an attempt to recanalise chronically occluded infrainguinal arteries using the MultiCross/CenterCross device at the St. John Hospital and Medical Center. An experienced operator with expertise in complex peripheral interventions performed all interventions. Informed consent was obtained from all patients for the procedure.

\section{CENTERCROSS AND MULTICROSS DEVICES}

The CenterCross device (Figure 1A) is designed to facilitate guidewire delivery through complex lesions, particularly through blockages in patients with PAD. The device comprises a resheathable, self-expandable nitinol scaffold that is $10 \mathrm{~mm}$ long and has an expanded diameter of $4 \mathrm{~mm}$. It has a $3 \mathrm{Fr}$ inner lumen, which accommodates either a 0.014 " or a 0.018 " microcatheter and guidewire, or a 0.035 " guidewire. The device has a working length of $130 \mathrm{~cm}$. The shaft diameter is $1.8 \mathrm{~mm}$ (0.071") which is compatible with a $7 \mathrm{Fr}$ guide catheter and a $6 \mathrm{Fr}$ introducer sheath. The device is advanced proximal to the lesion where the scaffold deploys to centre and anchor, providing maximum support for the microcatheter and guidewire to navigate through the lesion. Once the guidewire penetrates and crosses the lesion, CenterCross is removed from the vessel, allowing the operator to perform angioplasty, atherectomy or stenting of the lesion over the guidewire used to cross the lesion.

The MultiCross device (Figure 1B) is similar to the CenterCross, but enables three separate 0.014" guidewires to be delivered through a similar platform comprising a resheathable, self-expanding nitinol scaffold. These lumens are anchored away from the vessel wall to facilitate intraluminal access. It has a working length of $135 \mathrm{~cm}$ with a shaft diameter of $1.8 \mathrm{~mm}(0.071$ ") which is compatible with a $7 \mathrm{Fr}$ guide catheter and a $6 \mathrm{Fr}$ introducer sheath. The scaffold is $10 \mathrm{~mm}$ in length with an expanded diameter of $4.7 \mathrm{~mm}$. The MultiCross provides clinicians with greater options for wire escalation using multiple 0.014" guidewires simultaneously to cross the lesion. If the first guidewire meets resistance or is tracked into a subintimal plane, the operator may utilise a second or third 0.014 " guidewire in the remaining lumens. Once the lesion is crossed, the operator leaves the crossing guidewire in the distal vessel, resheaths the MultiCross and removes it from the vessel, allowing the operator to use the wire across the lesion to perform angioplasty, atherectomy or stenting of the lesion over the guidewire used to cross the lesion. These devices can be used to facilitate the treatment of any CTO in the peripheral arteries and there are no device-specific exclusions.

\section{PROCEDURAL DETAILS}

A contralateral retrograde or ipsilateral femoral access was obtained in patients with infrainguinal CTO. Intravenous unfractionated heparin was used to attain an activated clotting time $>250$ seconds. After attempts to use conventional wires to cross CTOs failed, CenterCross 

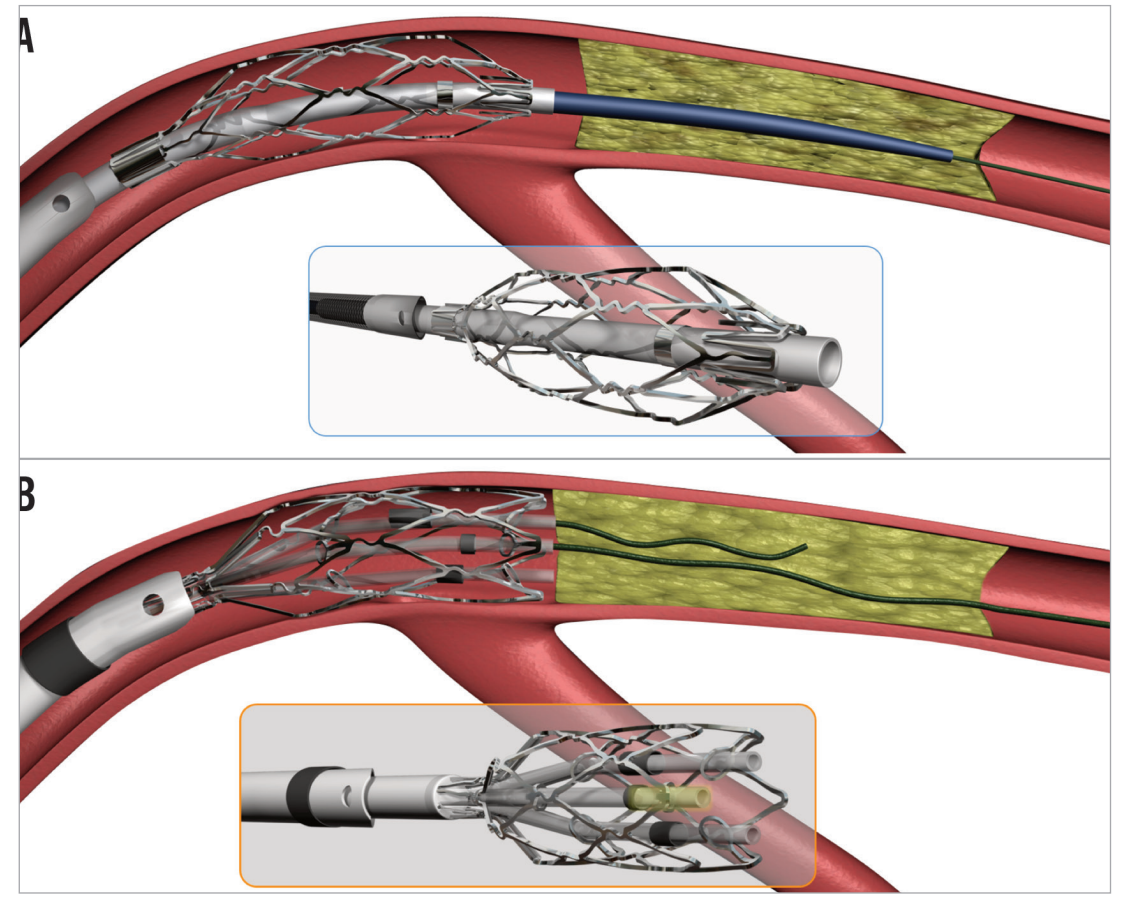

Figure 1. The CenterCross and MultiCross devices. A) Cartoon of the CenterCross device (Roxwood Medical, Inc.). B) Cartoon of the MultiCross device (Roxwood Medical, Inc.).

or MultiCross catheters were used to assist the advancement of a guidewire across the CTO. No other conventional CTO crossing devices were attempted before using CenterCross/MultiCross. CenterCross or MultiCross catheters were used to advance a guidewire across the CTO. Once this was achieved, various devices were used to treat the lesion including balloon angioplasty, directional or rotational atherectomy, drug-eluting balloon and/or stenting. Postdilation of lesions was performed in most cases. Atherectomy was performed with the use of an embolic protection device to prevent distal embolisation. Non-ionic contrast was used during all procedures. All patients received intravenous hydration before and after the procedure, aspirin $325 \mathrm{mg}$ before the procedure and thienopyridine after the procedure. Besides angiography, no other invasive imaging modality such as intravascular ultrasound was used before or after the intervention to guide and/or assess the results of the procedure. Groin closure was achieved using vascular closure devices or manual pressure. After the procedure, all patients were monitored overnight in a post-procedure observation unit and discharged the next day if stable.

\section{DATA COLLECTION AND DEFINITIONS}

Data were collected retrospectively for all patients and entered manually into an Excel database. The information gathered included patients' demographics, comorbid conditions, medical treatments, lesion characteristics, procedure details, in-hospital complications and outcomes. Procedure details included the success of crossing, crossing time (time from the insertion of the device until the lesion was crossed), total contrast used, fluoroscopy time and procedural success. Occlusion was defined as $100 \%$ blockage without notable antegrade blood flow during angiography. Lesion calcification was defined using a qualitative method as heavy (or not) if densities involved both sides of the arterial wall prior to contrast injection ${ }^{1}$. Successful device crossing was attained when advancement of the guidewire through the CTO lesion was verified by angiography. Procedural success was defined as $<30 \%$ residual stenosis in the absence of any dissection, perforation or distal embolisation. Bleeding was defined as a decrease in periprocedural haemoglobin of $>3 \mathrm{~g} / \mathrm{dl}$. Contrast-induced nephropathy was defined as a $25 \%$ increase in creatinine from baseline within 48 hours after the procedure. Mortality was all-cause death in-hospital. Bleeding risk at baseline for all individual patients was estimated using a previously published model ${ }^{8}$.

\section{STATISTICAL METHODS}

Continuous variables are expressed as mean \pm standard deviation and median and 25th and 75th percentile. Categorical variables are presented as frequency counts and percentages.

\section{Results}

Baseline demographics and comorbidities are shown in Table 1. Of the 53 patients with PAD who underwent CTO intervention, the majority were male (71\%). Patients' mean age was 74 years and there was a high prevalence of comorbid conditions, including hypertension and hyperlipidaemia in almost everyone, and diabetes and coronary artery disease in three quarters of the patients. The majority of patients were smokers (70\%). About three quarters of patients had had prior percutaneous peripheral vascular interventions, half had had prior peripheral bypass surgery and 
Table 1. Baseline demographics and comorbidities.

\section{Characteristics*}

No. of patients

53

Age, years (mean \pm SD)

Median (25th, 75th percentile), years

Gender, male

Race, white

Height, cm (mean \pm SD)

Median (25th, 75th percentile), cm

Weight, $\mathrm{kg}($ mean \pm SD)

Median (25th, 75th percentile), kg

Medical

history

Coronary artery disease

Hyperlipidaemia

Hypertension

Diabetes mellitus

Current smoker

Congestive heart failure

Atrial fibrillation

Stroke/transient ischaemic attack 15 (28.3\%)

Renal failure with dialysis

Chronic obstructive airway disease

$3(5.7 \%)$

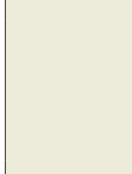

Bleeding risk

Peripheral vascular intervention

$12(22.6 \%)$

Peripheral bypass

$39(73.6 \%)$

Amputation

$24(45.3 \%)$

$14(26.4 \%)$

$16(30.2 \%)$

$25(47.2 \%)$

$7(13.2 \%)$

Indications

Intermediate

Low

Claudication

$24(45.3 \%)$

Critical limb ischaemia

$50(94.3 \%)$

Ankle-

brachial index

Toe-brachial

Right (mean \pm SD)

Left $($ mean \pm SD)

$0.88 \pm 0.34$

$0.82 \pm 0.28$

Right (mean $\pm S D)$

$0.50 \pm 0.22$

index

Left (mean \pm SD)

$0.50 \pm 0.17$

Pre-procedural creatinine, $\mathrm{mg} / \mathrm{dl}($ mean $\pm \mathrm{SD})$

$1.7 \pm 2.3$

Median (25th, 75th percentile), mg/dl

Post-procedural creatinine, $\mathrm{mg} / \mathrm{dl}$ (mean \pm SD)

$1.2(0.9,1.6)$

Median (25th, 75th percentile), mg/dl

$1.6 \pm 1.9$

Pre-procedural haemoglobin, g/dl (mean \pm SD)

$1.1(0.9,1.5)$

$11.5 \pm 1.9$

Median (25th, 75th percentile), g/dl

$12(10,13)$

Post-procedural haemoglobin, g/dl (mean \pm SD)

$10.4 \pm 1.9$

Median (25th, 75th percentile), g/dl

$$
\text { Aspirin }
$$

$11(9,12)$

\section{Clopidogrel}

$31(58.5 \%)$

Heparin

$42(79.2 \%)$

$53(100 \%)$

${ }^{*} n(\%)$ unless otherwise specified. SD: standard deviation

a quarter had had prior amputation. Almost all patients had symptoms at rest and over half presented with tissue loss.

Lesion location and characteristics are summarised in Table 2. CTO lesions in the popliteal artery and below were the most commonly treated vessels, comprising $89 \%$ of the total, whereas
Table 2. Angiographic features.

Characteristics*

\begin{tabular}{|l|l|c|}
\hline \multicolumn{2}{|l|}{ No. of patients } & 53 \\
\hline \multirow{3}{*}{$\begin{array}{l}\text { Lesion } \\
\text { location }\end{array}$} & Superficial femoral artery & $6(11.3 \%)$ \\
\cline { 2 - 3 } & Popliteal artery & $12(22.6 \%)$ \\
\cline { 2 - 3 } & Anterior tibial artery & $19(35.8 \%)$ \\
\cline { 2 - 3 } & Posterior tibial artery & $12(22.6 \%)$ \\
\cline { 2 - 3 } & Peroneal artery & $3(5.7 \%)$ \\
\cline { 2 - 3 } & Dorsalis pedis artery & $1(1.9 \%)$ \\
\hline Lesion length, mm (mean \pm SD) & $154.7 \pm 49.3$ \\
\hline \multicolumn{2}{|c|}{ Median (25th, 75th percentile), mm } & $3.4 \pm 1.2$ \\
\hline Vessel diameter, mm (mean \pm SD) & $3(3,5)$ \\
\hline \multicolumn{2}{|c|}{ Median (25th, 75th percentile), mm } \\
\hline *n (\%) unless otherwise specified. SD: standard deviation \\
\hline
\end{tabular}

superficial femoral artery occlusions represented the minority, comprising only $11 \%$ of the treated lesions. The mean length of CTO lesions was long with an average of $154.7 \pm 49.3 \mathrm{~mm}$. Heavy calcification was present in 21 of the 53 patients.

Procedure success was achieved in the vast majority of patients (92.4\%) (Table 3). This was achieved in $20 / 21$ patients $(95.2 \%)$ with heavy calcification versus $29 / 32$ patients (90.6\%) without heavy calcification. The average crossing and fluoroscopy times were 5.5 and 27 minutes, respectively, with a mean contrast medium use of $121 \mathrm{ml}$. After successful CTO lesion crossing, directional and rotational atherectomy were the most commonly performed procedures. Only a small minority $(n=8)$ of patients were treated with stent placement in our series.

\section{Table 3. Procedural outcomes.}

\section{Outcomes*}

\begin{tabular}{|c|c|c|}
\hline \multicolumn{2}{|l|}{ No. of patients } & 53 \\
\hline \multicolumn{2}{|l|}{ Crossing success } & 49 (92.4\%) \\
\hline \multicolumn{2}{|l|}{ Failure to cross } & $4(7.5 \%)$ \\
\hline \multicolumn{2}{|c|}{ Crossing time, $\min (\operatorname{mean} \pm \mathrm{SD})$} & $5.5 \pm 3.5$ \\
\hline \multicolumn{2}{|c|}{ Median (25th, 75th percentile), min } & $5(3,7)$ \\
\hline \multirow[t]{4}{*}{ Complications } & Distal embolisation & $1(1.9 \%)$ \\
\hline & Arterial perforation & $2(3.8 \%)$ \\
\hline & Arterial dissection & $0(0 \%)$ \\
\hline & Bleeding & $0(0 \%)$ \\
\hline \multicolumn{2}{|l|}{ Death } & $0(0 \%)$ \\
\hline \multicolumn{2}{|l|}{ Transfusion } & $0(0 \%)$ \\
\hline \multicolumn{2}{|c|}{ Contrast-induced nephropathy } & $0(0 \%)$ \\
\hline \multicolumn{2}{|c|}{ Fluoroscopy time, $\min (\operatorname{mean} \pm S D$ ) } & $26.9 \pm 14.4$ \\
\hline \multicolumn{2}{|c|}{ Median (25th, 75th percentile), min } & $25(17,32)$ \\
\hline \multicolumn{2}{|c|}{ Contrast used, $\mathrm{ml}($ mean $\pm \mathrm{SD}$ ) } & $120.6 \pm 48.7$ \\
\hline \multicolumn{2}{|c|}{ Median (25th, 75th percentile), ml } & $105(80,148)$ \\
\hline \multirow{4}{*}{$\begin{array}{l}\text { Treatment after } \\
\text { crossing chronic } \\
\text { total occlusion }\end{array}$} & Rotational atherectomy & 23 \\
\hline & Directional atherectomy & 19 \\
\hline & Balloon & 43 \\
\hline & Stent & 8 \\
\hline
\end{tabular}


Procedural complications were low (Table 3): only two patients had guidewire perforation and one had distal embolisation - these were managed conservatively without any major adverse consequences. Bleeding, need for transfusion, vessel dissection, contrast-induced nephropathy and/or need for emergent peripheral vascular surgery were not observed in any patients.

\section{Discussion}

We present the initial findings of novel crossing devices (CenterCross and MultiCross) for infrainguinal CTO lesions. These devices were designed to facilitate crossing by securing the guidewire just proximal to the CTO lesion. We must emphasise that this is a preliminary retrospective report of interventions upon only 53 patients with infrainguinal CTOs. The results were encouraging in this small group of patients with successful crossing attained in $92 \%$ of patients at a relatively short crossing time of 5.5 minutes and low contrast use (mean=121 ml) and average fluoroscopy time (mean=27 minutes), despite very long lesions (mean lesion length of $155 \pm 49.3 \mathrm{~mm}$ ). Additionally, qualitatively defined heavy calcification was not associated with device failure to cross the lesion. Finally, the procedures were completed with a low rate of complications (bleeding $0 \%$, dissection $0 \%$, perforation $3.8 \%$ and embolisation $1.9 \%$ ), and no patient required emergent peripheral arterial surgery.

Multiple other devices have been used for crossing CTO lesions with varying success rates ${ }^{1,2,6,9-16}$ (Table 4) and complication rates ${ }^{1,2,6,9-16}$ (Table 5). None of these devices was compared directly with another in a randomised fashion to evaluate their relative efficacy. Similarly, direct comparisons cannot be made between the CenterCross/MultiCross system and the other currently available devices reported in Table $\mathbf{4}$ and Table 5, as the patient populations, procedural details, and lesion characteristics may not be similar.
However, what can be stated is that the results of the current study are encouraging, demonstrating a relatively high procedural success rate of crossing lengthy lesions with a relatively short crossing time and contrast use with roughly comparable fluoroscopy times. Furthermore, it was encouraging that the current devices were safe and were associated with low complication rates, most of which were not life-threatening. Additionally, these favourable outcomes were achieved despite the fact that in our patients the vast majority of treated lesions were located in the popliteal artery and/or below this level (89\%); this contrasts with most other studies where the majority of treated CTO were superficial femoral artery lesions ${ }^{1,2,6,9-14}$. Available data suggest that popliteal or infrapopliteal CTO are associated with significantly lower success rates, longer procedural times and higher complication rates as compared to suprapopliteal lesions ${ }^{15}$.

Our data suggested that CenterCross/MultiCross might have the potential to improve procedural success without compromising safety in patients undergoing peripheral CTO interventions. Additionally, unlike many other devices, CenterCross/MultiCross catheters are easy to use, eliminating the need for ultrasound or other ancillary guidance, minimising cost in these patients.

\section{Limitations}

Our study should be viewed in the light of its limitations. The major limitation was that our investigation was a non-randomised small case series without a concurrent control arm, particularly comparing it directly with other devices for crossing CTO. The sample size was small to modest given preliminary experience. This was a single-centre study with a single experienced operator. Therefore, it does not represent broad contemporary clinical experience. MultiCross and CenterCross are relatively new devices and, while there may be a learning curve associated

Table 4. Comparison between different CTO crossing devices.

\begin{tabular}{|c|c|c|c|c|c|c|c|c|c|}
\hline Study & Device & N & $\begin{array}{c}\text { SFA } \\
\text { lesions }\end{array}$ & $\begin{array}{c}\text { Popliteal and } \\
\text { below }\end{array}$ & $\begin{array}{l}\text { Mean lesion } \\
\text { length (mm) }\end{array}$ & $\begin{array}{l}\text { Crossing } \\
\text { success }\end{array}$ & $\begin{array}{c}\text { Mean fluoro- } \\
\text { scopy time (min) }\end{array}$ & $\begin{array}{c}\text { Mean contrast } \\
(\mathrm{ml})\end{array}$ & $\begin{array}{l}\text { Mean crossing } \\
\text { time (min) }\end{array}$ \\
\hline CONNECT $^{9}$ & Wildcat & 88 & 75 (85.2\%) & $9(10.2 \%)$ & $174 \pm 96$ & $89.3 \%$ & 30.2 & 247.51 & $22.1 \pm 20.2$ \\
\hline PATRIOT $^{2}$ & Crosser & 85 & 52 (61.2\%) & $31(36.4 \%)$ & $129.6 \pm 78.7$ & $81.2 \%$ & $36.2 \pm 16.7$ & $242 \pm 113$ & $14.3 \pm 12.2$ \\
\hline Khalid et a ${ }^{10}$ & Crosser & 25 & 20 (74\%) & $10(37 \%)$ & $117 \pm 85$ & $41 \%(63 \%)^{\pi}$ & $\mathrm{N} / \mathrm{A}$ & $N / A$ & $\mathrm{~N} / \mathrm{A}$ \\
\hline Re0pen ${ }^{1}$ & TruePath & 85 & $61(71.8 \%)$ & $17(20 \%)$ & 166 & $80 \%$ & $23.9 \pm 14.7$ & $N / A$ & $8.2 \pm 9.8$ \\
\hline Banerjee et all ${ }^{11}$ & TruePath & 13 & 10 (76.9\%) & $3(23.1 \%)$ & $128.1 \pm 81$ & $76.9 \%$ & $41.1 \pm 18.3$ & $200 \pm 46.2$ & \\
\hline CONNECT II ${ }^{6}$ & Ocelot & 100 & $94(94 \%)$ & $4(4 \%)$ & $166 \pm 9.3$ & $72 \%(97 \%)^{\#}$ & $38.6 \pm 29.1$ & $223 \pm 144.7$ & $32.9 \pm 42.9$ \\
\hline Banerjee et al ${ }^{12}$ & Viance & 58 & 34 (58.6\%) & 24 (41.4\%) & $132 \pm 78$ & $87.9 \%$ & $39.1 \pm 21.2$ & $187.8 \pm 72$ & $\mathrm{~N} / \mathrm{A}$ \\
\hline Shetty et a ${ }^{13}$ & Frontrunner XP & 22 & 20 (90.9\%) & $2(9.1 \%)$ & $180 \pm 101$ & $95 \%$ & $N / A$ & N/A & $N / A$ \\
\hline Charalambous et al ${ }^{14}$ & Frontrunner XP & 26 & $26(100 \%)$ & $0(0 \%)$ & $172.6 \pm 62$ & $65.38 \%$ & 22.9 & N/A & 8 \\
\hline Sethi et al ${ }^{15}$ & Viance/CrossBoss & 29 & $0(0 \%)$ & $37(100 \%)$ & $81 \pm 64$ & $65 \%$ & $28 \pm 16$ & $189 \pm 72$ & $N / A$ \\
\hline XLPAD registry ${ }^{16}$ & Wire-catheter & 295 & $244(82.8 \%)$ & $51(17.2 \%)$ & $134.2 \pm 72.8$ & $51.9 \%$ & 36.8 & 167.7 & $N / A$ \\
\hline Current study & $\begin{array}{l}\text { CenterCross/ } \\
\text { MultiCross }\end{array}$ & 53 & $6(11.3 \%)$ & 47 (88.7\%) & 154.7 & $92.4 \%$ & 26.9 & 120.6 & 5.5 \\
\hline
\end{tabular}

"crossing CTO with use of Crosser was $41 \%$; with use of other CT0 crossing devices crossing success was $63 \%$. "crossing success with 0celot alone $72 \%$; crossing success with 0 celot plus assist device 97\%. N/A: not available; SFA: superficial femoral artery 
Table 5. Complications of different CTO crossing devices.

\begin{tabular}{|c|c|c|c|c|c|c|c|c|c|c|}
\hline Study & Device & $\mathbf{N}$ & Death & Bleeding & Transfusion & Perforation & Dissection & $\begin{array}{c}\text { Distal } \\
\text { embolisation }\end{array}$ & CIN & Other \\
\hline CONNECT $^{9}$ & Wildcat & 88 & 0 & * & * & $4(4.8 \%)$ & 0 & 0 & * & * \\
\hline PATRIOT $^{2}$ & Crosser & 85 & * & * & * & $14(16.4 \%)$ & * & * & * & $2(2.4 \%)^{\#}$ \\
\hline Khalid et al ${ }^{10}$ & Crosser & 25 & * & * & * & 5 & 1 & * & * & * \\
\hline ReOpen ${ }^{1}$ & TruePath & 85 & * & * & * & $20(23.5 \%)$ & * & * & * & $\begin{array}{c}24 \\
(28.2 \%)^{\pi}\end{array}$ \\
\hline Banerjee et $\mathrm{al}^{11}$ & TruePath & 13 & 0 & * & * & * & 2 & * & * & * \\
\hline CONNECT II ${ }^{6}$ & Ocelot & 100 & 0 & * & * & $2(2 \%)$ & 0 & 0 & * & * \\
\hline Banerjee et $\mathrm{al}^{12}$ & Viance & 58 & 0 & * & * & * & 0 & * & * & * \\
\hline Shetty et al ${ }^{13}$ & Frontrunner XP & 22 & * & * & * & * & * & * & * & * \\
\hline Charalambous et al ${ }^{14}$ & Frontrunner XP & 26 & * & * & * & 1 & 1 & 2 & * & * \\
\hline Sethi et al ${ }^{15}$ & Viance/CrossBoss & 29 & * & * & * & * & * & 1 & * & * \\
\hline XLPAD registry ${ }^{16}$ & Wire-catheter & 295 & * & * & * & * & * & * & * & * \\
\hline Current study & CenterCross/MultiCross & 53 & 0 & 0 & 0 & $2(3.8 \%)$ & 0 & $1(1.9 \%)$ & 0 & * \\
\hline
\end{tabular}

"limb loss. *not reported. "serious adverse events; majority were ischaemic event of contralateral limb requiring revascularisation. CIN: contrast-induced nephropathy

with their use, we were unable to provide insight into this. We did not routinely grade the severity of peripheral arterial calcification, a characteristic that may have important implications for crossing as well as for the success of intervention in CTO. Thus, we are unable to provide any information on the implications of the severity of peripheral arterial calcification on the success rate of crossing with the devices used in our study and on the eventual procedural success. The effectiveness/efficacy and safety of these new devices in broader contemporary practice and in comparison with other devices as well as their cost-effectiveness in peripheral CTO interventions needs to be evaluated in future studies in a large cohort of patients undergoing PAD interventions.

\section{Conclusions}

In conclusion, the present study demonstrates that MultiCross and CenterCross devices were effective and safe for the recanalisation of peripheral CTO lesions that were not amenable to conventional guidewires. Further study is required to define the role of these novel devices in the treatment of complex lesions, particularly CTOs in patients with PAD.

\section{Impact on daily practice}

Treating a CTO lesion remains challenging because of difficulty in crossing the lesion. Attempts to cross are associated with significant radiation, contrast use and longer procedure time. CenterCross/MultiCross devices are novel tools to cross CTO lesions. Our data revealed that CenterCross/MultiCross devices were effective in crossing CTO lesions in a short time with a relatively safe profile compared to other commonly used CTO crossing devices. Our data showed that using these novel devices may improve patient safety, comfort and healthcare costs and expands the armamentarium for the treatment of CTO.

\section{Funding}

The study was funded in part by modest grant support from Roxwood Medical, Inc., CA, USA.

The sponsors had no role in the study design, the analysis, the drafting and editing of the manuscript, the content of the final manuscript or the decision to publish these results.

\section{Conflict of interest statement}

T.P. Davis is on the advisory board for Roxwood Medical, Inc. The other authors have no conflicts of interest to declare.

\section{References}

1. Bosiers M, Diaz-Cartelle J, Scheinert D, Peeters P, Dawkins KD. Revascularization of lower extremity chronic total occlusions with a novel intraluminal recanalization device: results of the ReOpen study. J Endovasc Ther. 2014;21:61-70.

2. Laird J, Joye J, Sachdev N, Huang P, Caputo R, Mohiuddin I, Runyon J, Das T. Recanalization of infrainguinal chronic total occlusions with the crosser system: results of the PATRIOT trial. J Invasive Cardiol. 2014;26:497-504.

3. McKinsey JF, Goldstein L, Khan HU, Graham A, Rezeyat C, Morrissey NJ, Sambol E, Kent KC. Novel treatment of patients with lower extremity ischemia: use of percutaneous atherectomy in 579 lesions. Ann Surg. 2008;248:519-28.

4. Norgren L, Hiatt WR, Dormandy JA, Nehler MR, Harris KA, Fowkes FG; TASC II Working Group, Bell K, Caporusso J, Durand-Zaleski I, Komori K, Lammer J, Liapis C, Novo S, Razavi M, Robbs J, Schaper N, Shigematsu H, Sapoval M, White C, White J, Clement D, Creager M, Jaff M, Mohler E 3rd, Rutherford RB, Sheehan P, Sillesen H, Rosenfield K. InterSociety Consensus for the Management of Peripheral Arterial Disease (TASC II). Eur J Vasc Endovasc Surg. 2007;33 Suppl $1: \mathrm{S} 1-75$ 
5. Rogers J, Laird J. Overview of new technologies for lower extremity revascularization. Circulation. 2007;116:2072-85.

6. Selmon MR, Schwindt AG, Cawich IM, Chamberlin JR, Das TS, Davis TP, George JC, Janzer SF, Lopez LA, McDaniel HB, McKinsey JF, Pigott JP, Raja ML, Reimers B, Schreiber TL. Final results of the Chronic Total Occlusion Crossing With the Ocelot System II (CONNECT II) study. J Endovasc Ther. 2013;20: 770-81.

7. Davis T, Torey J. Options for Chronic Total Occlusions. A summary of the technologies that may enable treatment of difficult lesions. Endovascular Today. 2013;12:87-91.

8. Mehta SK, Frutkin AD, Lindsey JB, House JA, Spertus JA, Rao SV, Ou FS, Roe MT, Peterson ED, Marso SP; National Cardiovascular Data Registry. Bleeding in patients undergoing percutaneous coronary intervention: the development of a clinical risk algorithm from the National Cardiovascular Data Registry. Circ Cardiovasc Interv. 2009;2:222-9.

9. Pigott JP, Raja ML, Davis T; Connect Trial Investigators. A multicenter experience evaluating chronic total occlusion crossing with the Wildcat catheter (the CONNECT study). J Vasc Surg. 2012;56:1615-21.

10. Khalid MR, Khalid FR, Farooqui FA, Devireddy CM, Robertson GC, Niazi K. A novel catheter in patients with peripheral chronic total occlusions: a single center experience. Catheter Cardiovasc Interv. 2010;76:735-9.

11. Banerjee S, Sarode K, Das T, Hadidi O, Thomas R, Vinas A, Garg P, Mohammad A, Baig MS, Shammas NW, Brilakis ES. Endovascular treatment of infrainguinal chronic total occlusions using the TruePath device: features, handling, and 6-month outcomes. J Endovasc Ther. 2014;21:281-8.

12. Banerjee S, Thomas R, Sarode K, Mohammad A, Sethi S, Baig MS, Gigliotti OS, Ali MI, Klein A, Abu-Fadel MS, Shammas NW, Prasad A, Brilakis ES. Crossing of infrainguinal peripheral arterial chronic total occlusion with a blunt microdissection catheter. J Invasive Cardiol. 2014;26:363-9.

13. Shetty R, Vivek G, Thakkar A, Prasad R, Pai U, Nayak K. Safety and efficacy of the frontrunner XP catheter for recanalization of chronic total occlusion of the femoropopliteal arteries. J Invasive Cardiol. 2013;25:344-7.

14. Charalambous N, Schäfer PJ, Trentmann J, Hümme TH, Stöhring C, Müller-Hülsbeck S, Heller M, Jahnke T. Percutaneous intraluminal recanalization of long, chronic superficial femoral and popliteal occlusions using the Frontrunner XP CTO device: a single-center experience. Cardiovasc Intervent Radiol. 2010;33: 25-33.

15. Sethi S, Mohammad A, Ahmed SH, Germanwala S, Sarode K, Ortiz-Lopez C, Banerjee S, Prasad A. Recanalization of popliteal and infrapopliteal chronic total occlusions using Viance and CrossBoss crossing catheters: a multicenter experience from the XLPAD Registry. J Invasive Cardiol. 2015;27:2-7.

16. Sarode K, Mohammad A, Jelinek S, Mekala K, Banerjee A, Das S, Hadidi O, Mody P, Williams D, Klein A, Gigliotti O, Luna M, Addo T, Prasad A, Brilakis E, Banerjee S. Comparison of peripheral atrial chronic total occlusion crossing strategies in the XLPAD Registry. J Am Coll Cardiol. 2014;64(11_S). 